\title{
METHODS FOR DETERMINATION AND APPROXIMATION OF THE DOMAIN OF ATTRACTION IN THE CASE OF AUTONOMOUS DISCRETE DYNAMICAL SYSTEMS
}

\author{
ST. BALINT, E. KASLIK, A. M. BALINT, AND A. GRIGIS \\ Received 15 October 2004; Accepted 18 October 2004
}

A method for determination and two methods for approximation of the domain of attraction $D_{a}(0)$ of the asymptotically stable zero steady state of an autonomous, $\mathbb{R}$-analytical, discrete dynamical system are presented. The method of determination is based on the construction of a Lyapunov function $V$, whose domain of analyticity is $D_{a}(0)$. The first method of approximation uses a sequence of Lyapunov functions $V_{p}$, which converge to the Lyapunov function $V$ on $D_{a}(0)$. Each $V_{p}$ defines an estimate $N_{p}$ of $D_{a}(0)$. For any $x \in$ $D_{a}(0)$, there exists an estimate $N_{p^{x}}$ which contains $x$. The second method of approximation uses a ball $B(R) \subset D_{a}(0)$ which generates the sequence of estimates $M_{p}=f^{-p}(B(R))$. For any $x \in D_{a}(0)$, there exists an estimate $M_{p^{x}}$ which contains $x$. The cases $\left\|\partial_{0} f\right\|<1$ and $\rho\left(\partial_{0} f\right)<1 \leq\left\|\partial_{0} f\right\|$ are treated separately because significant differences occur.

Copyright @ 2006 Hindawi Publishing Corporation. All rights reserved.

\section{Introduction}

Let be the following discrete dynamical system:

$$
x_{k+1}=f\left(x_{k}\right) \quad k=0,1,2, \ldots,
$$

where $f: \Omega \rightarrow \Omega$ is an $\mathbb{R}$-analytic function defined on a domain $\Omega \subset \mathbb{R}^{n}, 0 \in \Omega$ and $f(0)=0$, that is, $x=0$ is a steady state (fixed point) of (1.1).

For $r>0$, denote by $B(r)=\left\{x \in \mathbb{R}^{n}:\|x\|<r\right\}$ the ball of radius $r$.

The steady state $x=0$ of $(1.1)$ is "stable" provided that given any ball $B(\varepsilon)$, there is a ball $B(\delta)$ such that if $x \in B(\delta)$ then $f^{k}(x) \in B(\varepsilon)$, for $k=0,1,2, \ldots$ [4].

If in addition there is a ball $B(r)$ such that $f^{k}(x) \rightarrow 0$ as $k \rightarrow \infty$ for all $x \in B(r)$ then the steady state $x=0$ is "asymptotically stable" [4].

The domain of attraction $D_{a}(0)$ of the asymptotically stable steady state $x=0$ is the set of initial states $x \in \Omega$ from which the system converges to the steady state itself, that is,

$$
D_{a}(0)=\left\{x \in \Omega \mid f^{k}(x) \stackrel{k \rightarrow \infty}{\longrightarrow} 0\right\}
$$

Hindawi Publishing Corporation Advances in Difference Equations Volume 2006, Article ID 23939, Pages 1-15

DOI 10.1155/ADE/2006/23939 
Theoretical research shows that the $D_{a}(0)$ and its boundary are complicated sets [5-9]. In most cases, they do not admit an explicit elementary representation. The domain of attraction of an asymptotically stable steady state of a discrete dynamical system is not necessarily connected (which is the case for continuous dynamical systems). This fact is shown by the following example.

Example 1.1. Let be the function $f: \mathbb{R} \rightarrow \mathbb{R}$ defined by $f(x)=(1 / 2) x-(1 / 4) x^{2}+(1 / 2) x^{3}+$ $(1 / 4) x^{4}$. The domain of attraction of the asymptotically stable steady state $x=0$ is $D_{a}(0)=$ $(-2.79,-2.46) \cup(-1,1)$ which is not connected.

Different procedures are used for the approximation of the $D_{a}(0)$ with domains having a simpler shape. For example, in the case of [4, Theorem 4.20, page 170] the domain which approximates the $D_{a}(0)$ is defined by a Lyapunov function $V$ built with the matrix $\partial_{0} f$ of the linearized system in 0 , under the assumption $\left\|\partial_{0} f\right\|<1$. In [2], a Lyapunov function $V$ is presented in the case when the matrix $\partial_{0} f$ is a contraction, that is, $\left\|\partial_{0} f\right\|<1$. The Lyapunov function $V$ is built using the whole nonlinear system, not only the matrix $\partial_{0} f . V$ is defined on the whole $D_{a}(0)$, and more, the $D_{a}(0)$ is the natural domain of analyticity of $V$. In [3], this result is extended for the more general case when $\rho\left(\partial_{0} f\right)<1$ (where $\rho\left(\partial_{0} f\right)$ denotes the spectral radius of $\partial_{0} f$.) This last result is the following.

Theorem 1.2 (see [3]). If the function $f$ satisfies the following conditions:

$$
\begin{gathered}
f(0)=0, \\
\rho\left(\partial_{0} f\right)<1,
\end{gathered}
$$

then 0 is an asymptotically stable steady state. $D_{a}(0)$ is an open subset of $\Omega$ and coincides with the natural domain of analyticity of the unique solution $V$ of the iterative first-order functional equation

$$
\begin{gathered}
V(f(x))-V(x)=-\|x\|^{2}, \\
V(0)=0 .
\end{gathered}
$$

The function $V$ is positive on $D_{a}(0)$ and $V(x) \stackrel{x \rightarrow x^{0}}{\rightarrow}+\infty$, for any $x^{0} \in \partial D_{a}(0),\left(\partial D_{a}(0) d e\right.$ notes the boundary of $\left.D_{a}(0)\right)$ or for $\|x\| \rightarrow \infty$.

The function $V$ is given by

$$
V(x)=\sum_{k=0}^{\infty}\left\|f^{k}(x)\right\|^{2} \quad \text { for any } x \in D_{a}(0) .
$$

The Lyapunov function $V$ can be found theoretically using relation (1.5). In the followings, we will shortly present the procedure of determination and approximation of the domain of attraction using the function $V$ presented in $[2,3]$.

The region of convergence $D_{0}$ of the power series development of $V$ in 0 is a part of the domain of attraction $D_{a}(0)$. If $D_{0}$ is strictly contained in $D_{a}(0)$, then there exists a point $x^{0} \in \partial D_{0}$ such that the function $V$ is bounded on a neighborhood of $x^{0}$. Let be the power 
series development of $V$ in $x^{0}$. The domain of convergence $D_{1}$ of the series centered in $x^{0}$ gives a new part $D_{1} \backslash\left(D_{0} \cap D_{1}\right)$ of the domain of attraction $D_{a}(0)$. At this step, the part $D_{0} \cup D_{1}$ of $D_{a}(0)$ is obtained.

If there exists a point $x^{1} \in \partial\left(D_{0} \cup D_{1}\right)$ such that the function $V$ is bounded on a neighborhood of $x^{1}$, then the domain $D_{0} \cup D_{1}$ is strictly included in the domain of attraction $D_{a}(0)$. In this case, the procedure described above is repeated in $x^{1}$.

The procedure cannot be continued in the case when it is found that on the boundary of the domain $D_{0} \cup D_{1} \cup \cdots \cup D_{p}$ obtained at step $p$, there are no points having neighborhoods on which $V$ is bounded.

This procedure gives an open connected estimate $D$ of the domain of attraction $D_{a}(0)$. Note that $f^{-k}(D), k \in \mathbb{N}$ is also an estimate of $D_{a}(0)$, which is not necessarily connected.

The procedure described above is illustrated by the following examples.

Example 1.3. Let be the $f: \mathbb{R} \rightarrow \mathbb{R}$ defined by $f(x)=x^{2}$. Due to the equality $f^{k}(x)=x^{2^{k}}$ the domain of attraction of the asymptotically stable steady state $x=0$ is $D_{a}(0)=(-1,1)$. The Lyapunov function is $V(x)=\sum_{k=0}^{\infty} x^{2^{k+1}}$. The domain of convergence of the series is $D_{0}=(-1,1)$ which coincides with $D_{a}(0)$.

Example 1.4. Let be the function $f: \Omega=(-\infty, 1) \rightarrow \Omega$ defined by $f(x)=x /(e+(1-e) x)$. Due to the equality $f^{k}(x)=x /\left(e^{k}+\left(1-e^{k}\right) x\right)$ the domain of attraction of the asymptotically stable steady state $x=0$ is $D_{a}(0)=(-\infty, 1)$. The power series expansion of the Lyapunov function $V(x)=\sum_{k=0}^{\infty}\left|f^{k}(x)\right|^{2}$ in 0 is

$$
V(x)=\sum_{m=2}^{\infty}(m-1) \sum_{k=0}^{\infty} e^{-2 k}\left(1-e^{-k}\right)^{m-2} x^{m}
$$

The radius of convergence of the series (1.6) is

$$
r_{0}=\lim _{m \rightarrow \infty} \sqrt[m]{(m-1) \sum_{k=0}^{\infty} e^{-2 k}\left(1-e^{-k}\right)^{m-2}}=1,
$$

therefore the domain of convergence of the series (1.6) is $D_{0}=(-1,1) \subset D_{a}(0)$. More, $V(x) \rightarrow \infty$ as $x \rightarrow 1$ and $V(-1)<\infty$. The radius of convergence of the power series expansion of $V$ in -1 is

$$
r_{-1}=\lim _{m \rightarrow \infty} \sqrt[m]{\sum_{k=1}^{\infty} \frac{e^{k}\left(e^{k}-1\right)^{m-2}\left[(m-3) e^{k}+2\right]}{\left(2 e^{k}-1\right)^{m+2}}}=1,
$$

therefore the domain of convergence of the power series development of $V$ in -1 is $D_{-1}=$ $(-2,0)$ which gives a new part of $D_{a}(0)$.

Numerical results for more complex examples are given in $[2,3]$. 
2. Theoretical results when the matrix $A=\partial_{0} f$ is a contraction (i.e., $\|A\|<1$ )

The function $f$ can be written as

$$
f(x)=A x+g(x) \text { for any } x \in \Omega,
$$

where $A=\partial_{0} f$ and $g: \Omega \rightarrow \Omega$ is an $\mathbb{R}$-analytic function such that $g(0)=0$ and $\lim _{x \rightarrow 0}(\|g(x)\|$ $/\|x\|)=0$.

Proposition 2.1. If $\|A\|<1$, then there exists $r>0$ such that $B(r) \subset \Omega$ and $\|f(x)\|<\|x\|$ for any $x \in B(r) \backslash\{0\}$.

Proof. Due to the fact that $\lim _{x \rightarrow 0}(\|g(x)\| /\|x\|)=0$ there exists $r>0$ such that $B(r) \subset \Omega$ and

$$
\|g(x)\|<(1-\|A\|)\|x\| \quad \text { for any } x \in B(r) \backslash\{0\} .
$$

Let be $x \in B(r) \backslash\{0\}$. Inequality (2.2) provides that

$$
\|f(x)\|=\|A x+g(x)\| \leq\|A\|\|x\|+\|g(x)\|<(\|A\|+1-\|A\|)\|x\|=\|x\|
$$

therefore, $\|f(x)\|<\|x\|$.

Definition 2.2. Let $R>0$ be the largest number such that $B(R) \subset \Omega$ and $\|f(x)\|<\|x\|$ for any $x \in B(R) \backslash\{0\}$.

If for any $r>0, B(r) \subset \Omega$ and $\|f(x)\|<\|x\|$ for any $x \in B(r) \backslash\{0\}$, then $R=+\infty$ and $B(R)=\Omega=\mathbb{R}^{n}$.

LEMMA 2.3. (a) $B(R)$ is invariant to the flow of system (1.1).

(b) For any $x \in B(R)$, the sequence $\left(\left\|f^{k}(x)\right\|\right)_{k \in \mathbb{N}}$ is decreasing.

(c) For any $p \geq 0$ and $x \in B(R) \backslash\{0\}, \Delta V_{p}(x)=V_{p}(f(x))-V_{p}(x)<0$, where

$$
V_{p}(x)=\sum_{k=0}^{p}\left\|f^{k}(x)\right\|^{2} \quad \text { for } x \in \Omega .
$$

Proof. (a) If $x=0$, then $f^{k}(0)=0$, for any $k \in \mathbb{N}$. For $x \in B(R) \backslash\{0\}$, we have $\|f(x)\|<$ $\|x\|$, which implies that $f(x) \in B(R)$, that is, $B(R)$ is invariant to the flow of system (1.1).

(b) By induction, it results that for $x \in B(R)$ we have $f^{k}(x) \in B(R)$ and $\left\|f^{k+1}(x)\right\| \leq$ $\left\|f^{k}(x)\right\|$, which means that the sequence $\left(\left\|f^{k}(x)\right\|\right)_{k \in \mathbb{N}}$ is decreasing.

(c) In particular, for $p \geq 0$ and $x \in B(R)$, we have $\left\|f^{p+1}(x)\right\| \leq\|f(x)\|<\|x\|$ and therefore, $\Delta V_{p}(x)=\left\|f^{p+1}(x)\right\|^{2}-\|x\|^{2}<0$.

Corollary 2.4. For any $p \geq 0$, there exists a maximal domain $G_{p} \subset \Omega$ such that $0 \in G_{p}$ and for $x \in G_{p} \backslash\{0\}$, the (positive definite) function $V_{p}$ verifies $\Delta V_{p}(x)<0$. In other words, for any $p \geq 0$, the function $V_{p}$ defined by (2.4) is a Lyapunov function for (1.1) on $G_{p}$. Moreover, $B(R) \subset G_{p}$ for any $p \geq 0$.

Theorem 2.5. $B(R)$ is an invariant set included in the domain of attraction $D_{a}(0)$.

Proof. Let be $x \in B(R) \backslash\{0\}$. We have to prove that $\lim _{k \rightarrow \infty} f^{k}(x)=0$. 
The sequence $\left(f^{k}(x)\right)_{k \in \mathbb{N}}$ is bounded: $f^{k}(x)$ belongs to $B(R)$. Let be $\left(f^{k_{j}}(x)\right)_{j \in \mathbb{N}}$ a convergent subsequence and let be $\lim _{j \rightarrow \infty} f^{k_{j}}(x)=y^{0}$. It is clear that $y^{0} \in B(R)$.

It can be shown that

$$
\left\|f^{k}(x)\right\| \geq\left\|y^{0}\right\| \quad \text { for any } k \in \mathbb{N} \text {. }
$$

For this, observe first that $f^{k_{j}}(x) \rightarrow y^{0}$ and $\left(\left\|f^{k_{j}}(x)\right\|\right)_{k \in \mathbb{N}}$ is decreasing (Lemma 2.3). These imply that $\left\|f^{k_{j}}(x)\right\| \geq\left\|y^{0}\right\|$ for any $k_{j}$. On the other hand, for any $k \in \mathbb{N}$, there exists $k_{j} \in \mathbb{N}$ such that $k_{j} \geq k$. Therefore, as the sequence $\left(\left\|f^{k}(x)\right\|\right)_{k \in \mathbb{N}}$ is decreasing (Lemma 2.3), we obtain that $\left\|f^{k}(x)\right\| \geq\left\|f^{k_{j}}(x)\right\| \geq\left\|y^{0}\right\|$.

We show now that $y^{0}=0$. Suppose the contrary, that is, $y^{0} \neq 0$.

Inequality (2.5) becomes

$$
\left\|f^{k}(x)\right\| \geq\left\|y^{0}\right\|>0 \quad \text { for any } k \in \mathbb{N} .
$$

By means of Lemma 2.3, we have that $\left\|f\left(y^{0}\right)\right\|<\left\|y^{0}\right\|$.

Therefore, there exists a neighborhood $U_{f\left(y^{0}\right)} \subset B(R)$ of $f\left(y^{0}\right)$ such that for any $z \in$ $U_{f\left(y^{0}\right)}$ we have $\|z\|<\left\|y^{0}\right\|$. On the other hand, for the neighborhood $U_{f\left(y^{0}\right)}$ there exists a neighborhood $U_{y^{0}} \subset B(R)$ of $y^{0}$ such that for any $y \in U_{y^{0}}$, we have $f(y) \in U_{f\left(y^{0}\right)}$. Therefore:

$$
\|f(y)\|<\left\|y^{0}\right\| \quad \text { for any } y \in U_{y^{0}} .
$$

As $f^{k_{j}}(x) \rightarrow y^{0}$, there exists $\bar{j}$ such that $f^{k_{j}}(x) \in U_{y^{0}}$, for any $j \geq \bar{j}$. Making $y=f^{k_{j}}(x)$ in (2.7), it results that

$$
\left\|f^{k_{j}+1}(x)\right\|=\left\|f\left(f^{k_{j}}(x)\right)\right\|<\left\|y^{0}\right\| \quad \text { for } j \geq \bar{j}
$$

which contradicts (2.6). This means that $y^{0}=0$, consequently, every convergent subsequence of $\left(f^{k}(x)\right)_{k \in \mathbb{N}}$ converges to 0 . This provides that the sequence $\left(f^{k}(x)\right)_{k \in \mathbb{N}}$ is convergent to 0 , and $x \in D_{a}(0)$.

Therefore, the ball $B(R)$ is contained in the domain of attraction of $D_{a}(0)$.

For $p \geq 0$ and $c>0$ let be $N_{p}^{c}$ the set

$$
N_{p}^{c}=\left\{x \in \Omega: V_{p}(x)<c\right\} .
$$

If $c=+\infty$, then $N_{p}^{c}=\Omega$.

Theorem 2.6. Let be $p \geq 0$. For any $c \in\left(0,(p+1) R^{2}\right]$, the set $N_{p}^{c}$ is included in the domain of attraction $D_{a}(0)$.

Proof. Let be $c \in\left(0,(p+1) R^{2}\right]$ and $x \in N_{p}^{c}$. Then $V_{p}(x)=\sum_{k=0}^{p}\left\|f^{k}(x)\right\|^{2}<c \leq(p+1) R^{2}$, therefore, there exists $k \in\{0,1, \ldots, p\}$ such that $\left\|f^{k}(x)\right\|^{2}<R^{2}$. It results that $f^{k}(x) \in$ $B(R) \subset D_{a}(0)$, therefore, $x \in D_{a}(0)$.

Remark 2.7. It is obvious that for $p \geq 0$ and $0<c^{\prime}<c^{\prime \prime}$ one has $N_{p}^{c^{\prime}} \subset N_{p}^{c^{\prime \prime}}$. Therefore, for a given $p \geq 0$, the largest part of $D_{a}(0)$ which can be found by this method is $N_{p}^{c_{p}}$, where 
$c_{p}=(p+1) R^{2}$. In the followings, we will use the notation $N_{p}$ instead of $N_{p}^{c_{p}}$. Shortly, $N_{p}=\left\{x \in \Omega: V_{p}(x)<(p+1) R^{2}\right\}$ is a part of $D_{a}(0)$. Let us note that $N_{0}=B(R)$.

Remark 2.8. If $R=+\infty$ (i.e., $\Omega=\mathbb{R}^{n}$ and $\|f(x)\|<\|x\|$, for any $x \in \mathbb{R} \backslash\{0\}$ ), then $N_{p}=$ $\mathbb{R}^{n}$ for any $p \geq 0$ and $D_{a}(0)=\mathbb{R}^{n}$.

Theorem 2.9. For the sets $\left(N_{p}\right)_{p \in \mathbb{N}}$, the following properties hold:

(a) for any $p \geq 0$, one has $N_{p} \subset N_{p+1}$;

(b) for any $p \geq 0$, the set $N_{p}$ is invariant to $f$;

(c) for any $x \in D_{a}(0)$, there exists $p^{x} \geq 0$ such that $x \in N_{p^{x}}$.

Proof. (a) Let be $p \geq 0$ and $x \in N_{p}$. Then $V_{p}(x)=\sum_{k=0}^{p}\left\|f^{k}(x)\right\|^{2}<(p+1) R^{2}$, therefore, there exists $k \in\{0,1, \ldots, p\}$ such that $\left\|f^{k}(x)\right\|^{2}<R^{2}$. It results that $f^{k}(x) \in B(R)$ and therefore $f^{m}(x) \in B(R)$, for any $m \geq k$. For $m=p+1$ we obtain $\left\|f^{p+1}(x)\right\|<R$, hence $V_{p+1}(x)=V_{p}(x)+\left\|f^{p+1}(x)\right\|^{2}<(p+1) R^{2}+R^{2}=(p+2) R^{2}$. Therefore, $x \in N_{p+1}$.

(b) Let be $x \in N_{p}$. If $\|x\|<R$ then $\left\|f^{m}(x)\right\|<R$ for any $m \geq 0$ (by means of Lemma 2.3). This implies that $V_{p}(f(x))=\sum_{k=0}^{p}\left\|f^{k}(f(x))\right\|^{2}=\sum_{k=1}^{p+1}\left\|f^{k}(x)\right\|^{2}<(p+$ 1) $R^{2}$, meaning that $f(x) \in N_{p}$.

Let us suppose that $\|x\| \geq R$. As $x \in N_{p}$, we have that $V_{p}(x)=\sum_{k=0}^{p}\left\|f^{k}(x)\right\|^{2}<(p+$ 1) $R^{2}$, therefore, there exists $k \in\{0,1, \ldots, p\}$ such that $\left\|f^{k}(x)\right\|<R$. It results that $f^{k}(x) \in$ $B(R)$ and therefore $f^{m}(x) \in B(R)$, for any $m \geq k$. For $m=p+1$ we obtain $\left\|f^{p+1}(x)\right\|<R$. This implies that

$$
V_{p}(f(x))=V_{p}(x)+\left\|f^{p+1}(x)\right\|^{2}-\|x\|^{2}<(p+1) R^{2}+R^{2}-R^{2}=(p+1) R^{2}
$$

therefore $f(x) \in N_{p}$.

(c) Suppose the contrary, that is, there exist $x \in D_{a}(0)$ such that for any $p \geq 0, x \notin$ $N_{p}$. Therefore, $V_{p}(x) \geq(p+1) R^{2}$ for any $p \geq 0$. Passing to the limit for $p \rightarrow \infty$ in this inequality, provides that $V(x)=\infty$. This means $x \in \partial D_{a}(0)$ which contradicts the fact that $x$ belongs to the open set $D_{a}(0)$. In conclusion, there exists $p^{x} \geq 0$ such that $x \in$ $N_{p^{x}}$.

For $p \geq 0$ let be $M_{p}=f^{-p}(B(R))=\left\{x \in \Omega: f^{p}(x) \in B(R)\right\}$, obtained by the trajectory reversing method.

Theorem 2.10. The following properties hold:

(a) $M_{p} \subset D_{a}(0)$ for any $p \geq 0$;

(b) for any $p \geq 0, M_{p}$ is invariant to $f$;

(c) $M_{p} \subset M_{p+1}$ for any $p \geq 0$;

(d) for any $x \in D_{a}(0)$, there exists $p^{x} \geq 0$ such that $x \in M_{p^{x}}$.

Proof. (a) As $M_{p}=f^{-P}(B(R))$ and $B(R) \subset D_{a}(0)$ (see Theorem 2.5) it is clear that $M_{p} \subset$ $D_{a}(0)$.

(b) and (c) follow easily by induction, using Lemma 2.3 .

(d) $x \in D_{a}(0)$ provides that $f^{p}(x) \rightarrow 0$ as $p \rightarrow \infty$. Therefore, there exists $p^{x} \in \mathbb{N}$ such that $f^{p}(x) \in B(R)$, for any $p \geq p^{x}$. This provides that $x \in M_{p}$ for any $p \geq p^{x}$. 
Both sequences of sets $\left(M_{p}\right)_{p \in \mathbb{N}}$ and $\left(N_{p}\right)_{p \in \mathbb{N}}$ are increasing, and are made up of estimates of $D_{a}(0)$. From the practical point of view, it is important to know which sequence converges more quickly. The next theorem provides that the sequence $\left(M_{p}\right)_{p \in \mathbb{N}}$ converges more quickly than $\left(N_{p}\right)_{p \in \mathbb{N}}$, meaning that for $p \geq 0$, the set $M_{p}$ is a larger estimate of $D_{a}(0)$ then $N_{p}$.

Theorem 2.11. For any $p \geq 0$, one has $N_{p} \subset M_{p}$.

Proof. Let be $p \geq 0$ and $x \in N_{p}$. We have that $V_{p}(x)=\sum_{k=0}^{p}\left\|f^{k}(x)\right\|^{2}<(p+1) R^{2}$, therefore, there exists $k \in\{0,1, \ldots, p\}$ such that $\left\|f^{k}(x)\right\|<R$. This implies that $f^{m}(x) \in B(R)$, for any $m \geq k$. For $m=p$ we obtain $f^{p}(x) \in B(R)$, meaning that $x \in M_{p}$.

\section{Theoretical results when $A=\partial_{0} f$ is a convergent noncontractive matrix}

(i.e., $\rho(A)<1 \leq\|A\|)$

Proposition 3.1. If $\rho(A)<1 \leq\|A\|$, then there exist $\tilde{p} \geq 2$ and $r_{\tilde{p}}>0$ such that $B\left(r_{\tilde{p}}\right) \subset \Omega$ and $\left\|f^{p}(x)\right\|<\|x\|$ for any $p \in\{\tilde{p}, \tilde{p}+1, \ldots, 2 \tilde{p}-1\}$ and $x \in B\left(r_{\tilde{p}}\right) \backslash\{0\}$.

Proof. We have that $\rho(A)<1$ if and only if $\lim _{p \rightarrow \infty} A^{p}=0$ (see [1]), which provides (together with $\|A\| \geq 1$ ) that there exists $\tilde{p} \geq 2$ such that $\left\|A^{p}\right\|<1$ for any $p \geq \tilde{p}$. Let be $\tilde{p} \geq 2$ fixed with this property.

The formula of variation of constants for any $p$ gives:

$$
f^{p}(x)=A^{p} x+\sum_{k=0}^{p-1} A^{p-k-1} g\left(f^{k}(x)\right) \quad \forall x \in \Omega, p \in \mathbb{N}^{\star} .
$$

Due to the fact that for any $k \in \mathbb{N}$ we have $\lim _{x \rightarrow 0}\left(\left\|g\left(f^{k}(x)\right)\right\| /\|x\|\right)=0$, there exists $r_{\tilde{p}}>0$ such that for any $p \in\{\tilde{p}, \tilde{p}+1, \ldots, 2 \tilde{p}-1\}$ the following inequality holds:

$$
\sum_{k=0}^{p-1}\left\|A^{p-k-1}\right\|\left\|\mid g\left(f^{k}(x)\right)\right\|<\left(1-\left\|A^{p}\right\|\right)\|x\| \quad \text { for } x \in B\left(r_{\tilde{p}}\right) \backslash\{0\} .
$$

Let be $x \in B\left(r_{\tilde{p}}\right) \backslash\{0\}$ and $p \in\{\tilde{p}, \tilde{p}+1, \ldots, 2 \tilde{p}-1\}$. Using (3.1) and (3.2) we have

$$
\begin{aligned}
\left\|f^{p}(x)\right\| & =\left\|A^{p} x+\sum_{k=0}^{p-1} A^{p-k-1} g\left(f^{k}(x)\right)\right\| \\
& \leq\left\|A^{p}\right\|\|x\|+\sum_{k=0}^{p-1}\left\|A^{p-k-1}\right\|\left\|g\left(f^{k}(x)\right)\right\| \\
& <\left(\left\|A^{p}\right\|+1-\left\|A^{p}\right\|\right)\|x\|=\|x\| .
\end{aligned}
$$

Therefore, $\left\|f^{p}(x)\right\|<\|x\|$ for $p \in\{\tilde{p}, \tilde{p}+1, \ldots, 2 \tilde{p}-1\}$ and $x \in B\left(r_{\tilde{p}}\right) \backslash\{0\}$.

Definition 3.2. Let $\tilde{p} \geq 2$ be the smallest number such that $\left\|A^{p}\right\|<1$ for any $p \geq \tilde{p}$ (see the proof of Proposition 3.1). Let $\widetilde{R}>0$ the largest number be such that $B(\widetilde{R}) \subset \Omega$ and $\left\|f^{p}(x)\right\|<\|x\|$ for $p \in\{\tilde{p}, \tilde{p}+1, \ldots, 2 \tilde{p}-1\}$ and $x \in B(\widetilde{R}) \backslash\{0\}$. 
If for any $r>0$, we have that $B(r) \subset \Omega$ and $\left\|f^{p}(x)\right\|<\|x\|$ for any $p \in\{\tilde{p}, \tilde{p}+1, \ldots$, $2 \tilde{p}-1\}$ and $x \in B(r) \backslash\{0\}$, then $\widetilde{R}=+\infty$ and $B(\widetilde{R})=\Omega=\mathbb{R}^{n}$.

Lemma 3.3. (a) For any $x \in B(\tilde{R})$ and $p \in\{\tilde{p}, \tilde{p}+1, \ldots, 2 \tilde{p}-1\}$, the sequence $\left(\left\|f^{k p}(x)\right\|\right)_{k \in \mathbb{N}}$ is decreasing.

(b) For any $p \geq \tilde{p}$ and $x \in B(\tilde{R}) \backslash\{0\},\|f p(x)\|<\|x\|$.

(c) For any $p \geq \tilde{p}$ and $x \in B(\widetilde{R}) \backslash\{0\}, \Delta V_{p}(x)=V_{p}(f(x))-V_{p}(x)<0$, where $V_{p}$ is defined by (2.4).

Proof. (a) If $x=0$, then $f^{p}(0)=0$, for any $p \geq 0$.

Let be $x \in B(\widetilde{R}) \backslash\{0\}$. We know that $\left\|f^{p}(x)\right\|<\|x\|$ for any $p \in\{\tilde{p}, \tilde{p}+1, \ldots, 2 \tilde{p}-1\}$. It results that $f^{p}(x) \in B(\widetilde{R})$ for any $p \in\{\tilde{p}, \tilde{p}+1, \ldots, 2 \tilde{p}-1\}$. This implies that for any $k \in \mathbb{N}^{\star}$ we have $\left\|f^{k p}(x)\right\|<\|x\|$ and $\left\|f^{(k+1) p}(x)\right\| \leq\left\|f^{k p}(x)\right\|$, meaning that the sequence $\left(\left\|f^{k p}(x)\right\|\right)_{k \in \mathbb{N}}$ is decreasing.

(b) Let be $x \in B(\widetilde{R}) \backslash\{0\}$. Inequality $\|f p(x)\|<\|x\|$ is true for any $p \in\{\tilde{p}, \tilde{p}+1, \ldots$, $2 \tilde{p}-1\}$.

Let be $p \geq 2 \tilde{p}$. There exists $q \in \mathbb{N}^{\star}$ and $p^{\prime} \in\{\tilde{p}, \tilde{p}+1, \ldots, 2 \tilde{p}-1\}$ such that $p=q \tilde{p}+$ $p^{\prime}$. Using (a), we have that $f^{p^{\prime}}(x) \in B(\tilde{R})$ and $f q \tilde{p}(y) \leq\|y\|$, for any $y \in B(\tilde{R})$, therefore

$$
\left\|f^{p}(x)\right\|=\left\|f^{q \tilde{p}}\left(f^{p^{\prime}}(x)\right)\right\| \leq\left\|f^{p^{\prime}}(x)\right\|<\|x\|
$$

(c) results directly from (b).

Corollary 3.4. For any $p \geq \tilde{p}$, there exists a maximal domain $G_{p} \subset \Omega$ such that $0 \in G_{p}$ and for any $x \in G_{p} \backslash\{0\}$, the (positive definite) function $V_{p}$ verifies $\Delta V_{p}(x)<0$. In other words, for any $p \geq \tilde{p}$, the function $V_{p}$ is a Lyapunov function for (1.1) on $G_{p}$. More, $B(\widetilde{R}) \subset$ $G_{p}$ for any $p \geq \tilde{p}$.

Lemma 3.5. For any $k \geq \tilde{p}$, there exists $q_{k} \in \mathbb{N}$ such that

$$
\left\|f^{\left(q_{k}+3\right)} \tilde{p}(x)\right\| \leq\left\|f^{k}(x)\right\| \leq\left\|f^{q_{k} \tilde{p}}(x)\right\| \quad \text { for any } x \in B(\widetilde{R}) .
$$

Proof. Let be $k \geq \tilde{p}$. There exists a unique $q_{k} \in \mathbb{N}$ and a unique $p_{k} \in\{\tilde{p}, \tilde{p}+1, \ldots, 2 \tilde{p}-1\}$ such that $k=q_{k} \tilde{p}+p_{k}$. Lemma 3.3 provides that for any $x \in B(\widetilde{R})$ we have that $f_{k} \tilde{p}(x) \in$ $B(\widetilde{R})$ and $\left\|f^{p_{k}}(x)\right\| \leq\|x\|$. It results that

$$
\left\|f^{k}(x)\right\|=\left\|f^{p_{k}}\left(f^{q_{k} \tilde{p}}(x)\right)\right\| \leq\left\|f^{q_{k} \tilde{p}}(x)\right\| \quad \text { for any } x \in B(\bar{R}) .
$$

On the other hand, we have $\left(q_{k}+3\right) \tilde{p}=k+\left(3 \tilde{p}-p_{k}\right)$. As $\left(3 \tilde{p}-p_{k}\right) \in\{\tilde{p}+1, \tilde{p}+2, \ldots, 2 \tilde{p}\}$ and $k \geq \tilde{p}$, Lemma 3.3 provides that for any $x \in B(\widetilde{R})$ we have that $f^{k}(x) \in B(\tilde{R})$ and 
$\left\|f^{3 \tilde{p}-p_{k}}(x)\right\| \leq\|x\|$. Therefore

$$
\left\|f^{\left(q_{k}+3\right) \tilde{p}}(x)\right\|=\left\|f^{3 \tilde{p}-p_{k}}\left(f^{k}(x)\right)\right\| \leq\left\|f^{k}(x)\right\| \quad \text { for any } x \in B(\widetilde{R}) .
$$

Combining the two inequalities, we get that

$$
\left\|f^{\left(q_{k}+3\right)} \tilde{p}(x)\right\| \leq\left\|f^{k}(x)\right\| \leq\left\|f^{q_{k} \tilde{p}}(x)\right\| \quad \text { for any } x \in B(\widetilde{R})
$$

which concludes the proof.

Theorem 3.6. $B(\widetilde{R})$ is included in the domain of attraction $D_{a}(0)$.

Proof. Let be $x \in B(\tilde{R}) \backslash\{0\}$. We have to prove that $\lim _{k \rightarrow \infty} f^{k}(x)=0$.

The sequence $\left(f^{k}(x)\right)_{k \in \mathbb{N}}$ is bounded (see Lemma 3.3). Let be $\left(f^{k_{j}}(x)\right)_{j \in \mathbb{N}}$ a convergent subsequence and let be $\lim _{j \rightarrow \infty} f^{k_{j}}(x)=y^{0}$.

We suppose, without loss of generality, that $k_{j} \geq \tilde{p}$ for any $j \in \mathbb{N}$. Lemma 3.5 provides that for any $j \in \mathbb{N}$ there exists $q_{j} \in \mathbb{N}$ such that

$$
\left\|f^{\left(q_{j}+3\right) \tilde{p}}(x)\right\| \leq\left\|f^{k_{j}}(x)\right\| \leq\left\|f^{q_{j} \tilde{p}}(x)\right\|
$$

As $\left(\left\|f^{q_{j} \tilde{p}}(x)\right\|\right)_{j \in \mathbb{N}}$ and $\left(\left\|f^{\left(q_{j}+3\right)} \tilde{p}(x)\right\|\right)_{j \in \mathbb{N}}$ are subsequences of the convergent sequence $(\|f q \tilde{p}(x)\|)_{q \in \mathbb{N}}$ (decreasing, according to Lemma 3.3), it results that they are convergent. The double inequality (3.9) provides that $\lim _{j \rightarrow \infty}\left\|f^{q_{j}} \tilde{p}(x)\right\|=\left\|y^{0}\right\|$. Therefore, $\lim _{q \rightarrow \infty}$ $\|f \widetilde{p}(x)\|=\left\|y^{0}\right\|$.

It can be shown that

$$
\left\|f^{k}(x)\right\| \geq\left\|y^{0}\right\| \text { for any } k \geq \tilde{p}
$$

For this, remark that $\lim _{q \rightarrow \infty}\|f q \tilde{p}(x)\|=\left\|y^{0}\right\|$ and $(\|f q \tilde{p}(x)\|)_{q \in \mathbb{N}}$ is decreasing (Lemma 3.3), which implies that $\|f q \tilde{p}(x)\| \geq\left\|y^{0}\right\|$ for any $q \in \mathbb{N}$. On the other hand, Lemma 3.5 provides that for any $k \geq \tilde{p}$ there exists $q_{k}$ such that $\left\|f^{\left(q_{k}+3\right)} \tilde{p}(x)\right\| \leq\left\|f^{k}(x)\right\|$. Therefore, $\left\|f^{k}(x)\right\| \geq\left\|f^{\left(q_{k}+3\right) \tilde{p}}(x)\right\| \geq\left\|y^{0}\right\|$, for any $k \geq \tilde{p}$.

We show now that $y^{0}=0$. Suppose the contrary, that is, $y^{0} \neq 0$.

Inequality (3.10) becomes

$$
\left\|f^{k}(x)\right\| \geq\left\|y^{0}\right\|>0 \quad \text { for any } k \geq \tilde{p}
$$

By means of Lemma 3.3, we have that $\left\|f \tilde{p}\left(y^{0}\right)\right\|<\left\|y^{0}\right\|$.

There exists a neighborhood $U_{f \tilde{p}\left(y^{0}\right)} \subset B(\widetilde{R})$ of $f \widetilde{p}\left(y^{0}\right)$ such that for any $z \in U_{f \widetilde{p}\left(y^{0}\right)}$ we have $\|z\|<\left\|y^{0}\right\|$. On the other hand, for the neighborhood $U_{f^{\tilde{p}}\left(y^{0}\right)}$ there exists a neighborhood $U_{y^{0}} \subset B(\tilde{R})$ of $y^{0}$ such that for any $y \in U_{y^{0}}$, we have $f \widetilde{p}(y) \in U_{f^{\tilde{p}}\left(y^{0}\right)}$. Therefore:

$$
\left\|f^{\tilde{p}}(y)\right\|<\left\|y^{0}\right\| \quad \text { for any } y \in U_{y^{0}} .
$$


As $f^{k_{j}}(x) \rightarrow y^{0}$, there exists $\bar{j}$ such that $f^{k_{j}}(x) \in U_{y^{0}}$, for any $j \geq \bar{j}$. Making $y=f^{k_{j}}(x)$ in (3.12), it results that

$$
\left\|f^{k_{j}+\tilde{p}}(x)\right\|=\left\|f^{\tilde{p}}\left(f^{k_{j}}(x)\right)\right\|<\left\|y^{0}\right\| \quad \text { for } j \geq \bar{j}
$$

which contradicts (3.11). This means that $y^{0}=0$, consequently, every convergent subsequence of $\left(f^{k}(x)\right)_{k \in \mathbb{N}}$ converges to 0 . This provides that the sequence $\left(f^{k}(x)\right)_{k \in \mathbb{N}}$ is convergent to 0 , and $x \in D_{a}(0)$.

Therefore, the ball $B(\widetilde{R})$ is contained in the domain of attraction of $D_{a}(0)$.

Theorem 3.7. Let be $p \geq 0$. For any $c \in\left(0,(p+1) \widetilde{R}^{2}\right]$, the set $N_{p}^{c}$ is included in the domain of attraction $D_{a}(0)$.

Proof. Let be $c \in\left(0,(p+1) \tilde{R}^{2}\right]$ and $x \in N_{p}^{c}$. Then $V_{p}(x)=\sum_{k=0}^{p}\left\|f^{k}(x)\right\|^{2}<c \leq(p+1) \tilde{R}^{2}$, therefore, there exists $k \in\{0,1, \ldots, p\}$ such that $\left\|f^{k}(x)\right\|^{2}<\widetilde{R}^{2}$. It results that $f^{k}(x) \in$ $B(\widetilde{R}) \subset D_{a}(0)$, therefore, $x \in D_{a}(0)$.

Remark 3.8. It is obvious that for $p \geq 0$ and $0<c^{\prime}<c^{\prime \prime}$ one has $N_{p}^{c^{\prime}} \subset N_{p}^{c^{\prime \prime}}$. Therefore, for a given $p \geq 0$, the largest part of $D_{a}(0)$ which can be found by this method is $N_{p}^{\tilde{c}_{p}}$, where $\tilde{c}_{p}=(p+1) \widetilde{R}^{2}$. In the followings, we will use the notation $\tilde{N}_{p}$ instead of $N_{p}^{\widetilde{c}_{p}}$. Shortly, $\widetilde{N}_{p}=\left\{x \in \Omega: V_{p}(x)<(p+1) \widetilde{R}^{2}\right\}$ is a part of $D_{a}(0)$. Let us note that $\tilde{N}_{0}=B(\widetilde{R})$.

Remark 3.9. If $\widetilde{R}=+\infty$ (i.e., $\Omega=\mathbb{R}^{n}$ and $\left\|f^{p}(x)\right\|<\|x\|$, for any $p \in\{\tilde{p}, \tilde{p}+1, \ldots, 2 \tilde{p}-1\}$ and $x \in \mathbb{R} \backslash\{0\})$, then $\tilde{N}_{p}=\mathbb{R}^{n}$ for any $p \geq 0$ and $D_{a}(0)=\mathbb{R}^{n}$.

Theorem 3.10. For any $x \in D_{a}(0)$ there exists $p^{x} \geq 0$ such that $x \in \tilde{N}_{p^{x}}$.

Proof. Let be $x \in D_{a}(0)$. Suppose the contrary, that is, $x \notin \tilde{N}_{p}$ for any $p \geq 0$. Therefore, $V_{p}(x) \geq(p+1) \widetilde{R}^{2}$ for any $p \geq 0$. Passing to the limit when $p \rightarrow \infty$ in this inequality provides that $V(x)=\infty$. This means $x \in \partial D_{a}(0)$ which contradicts the fact that $x$ belongs to the open set $D_{a}(0)$. In conclusion, there exists $p^{x} \geq 0$ such that $x \in \tilde{N}_{p^{x}}$.

Remark 3.11. The sequence of sets $\left(\tilde{N}_{p}\right)_{p \in \mathbb{N}}$ is generally not increasing (see Section 4: Numerical examples, the Van der Pol equation).

Open question. Is the sequence of sets $\left(\tilde{N}_{p}\right)_{p \geq \tilde{p}}$ increasing?

For $p \geq 0$ let be $\widetilde{M}_{p}=f^{-p}(B(\widetilde{R}))=\left\{x \in \Omega: f^{p}(x) \in B(\widetilde{R})\right\}$, obtained by the trajectory reversing method.

Theorem 3.12. For the sets $\left(\widetilde{M}_{p}\right)_{p \in \mathbb{N}}$, the following properties hold:

(a) $\widetilde{M}_{p} \subset D_{a}(0)$, for any $p \geq 0$;

(b) $\widetilde{M}_{k p} \subset \widetilde{M}_{(k+1) p}$ for any $k \in \mathbb{N}$ and $p \in\{\tilde{p}, \tilde{p}+1, \ldots, 2 \tilde{p}-1\}$;

(c) for any $x \in D_{a}(0)$, there exists $p^{x} \geq 0$ such that $x \in \widetilde{M}_{p^{x}}$.

Proof. (a) As $\widetilde{M}_{p}=f^{-p}(B(\widetilde{R}))$ and $B(\widetilde{R}) \subset D_{a}(0)$ (see Theorem 3.6) it is clear that $\widetilde{M}_{p} \subset$ $D_{a}(0)$.

(b) follows easily by induction, using Lemma 3.3.

(c) $x \in D_{a}(0)$ provides that $f^{p}(x) \rightarrow 0$ as $p \rightarrow \infty$. Therefore, there exists $p^{x} \geq 0$ such that $f^{p}(x) \in B(\widetilde{R})$, for any $p \geq p^{x}$. This provides that $x \in \widetilde{M}_{p}$ for any $p \geq p^{x}$. 
St. Balint et al. 11

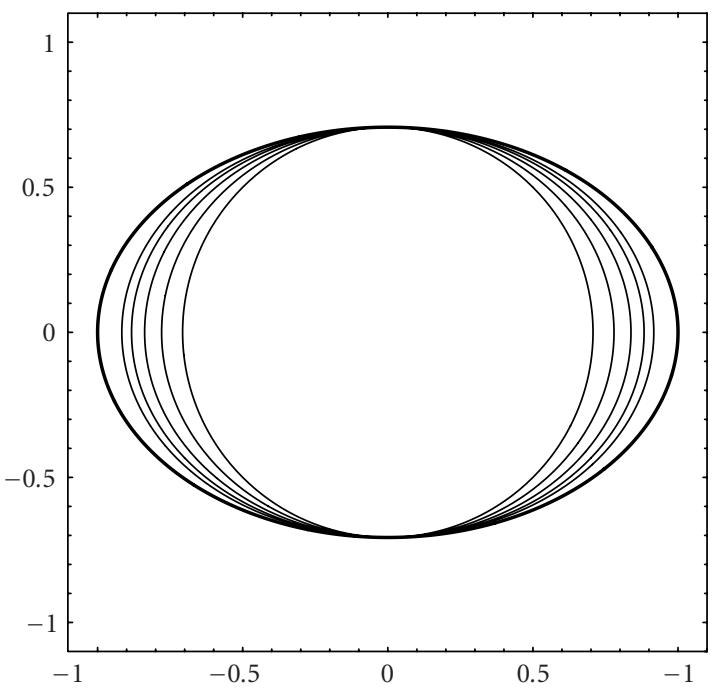

Figure 4.1. The sets $N_{p}, p=\overline{0,4}$ and $\partial D_{a}(0,0)$ for $(4.1)$.

Remark 3.13. The sequence of sets $\left(\widetilde{M}_{p}\right)_{p \in \mathbb{N}}$ is generally not increasing (see Section 4: Numerical examples, the Van der Pol equation).

Both sequences of sets $\left(\widetilde{M}_{p}\right)_{p \in \mathbb{N}}$ and $\left(\tilde{N}_{p}\right)_{p \in \mathbb{N}}$ are made up of estimates of $D_{a}(0)$. From the practical point of view, it would be important to know which one of the sets $\widetilde{M}_{p}$ or $\tilde{N}_{p}$ is a larger estimate of $D_{a}(0)$ for a fixed $p \geq \tilde{p}$. Such result could not be established, but the following theorem holds.

Theorem 3.14. For any $p \geq 0$, one has $\tilde{N}_{p} \subset \widetilde{M}_{p+\tilde{p}}$.

Proof. Let be $p \geq 0$ and $x \in \tilde{N}_{p}$. We have that $V_{p}(x)=\sum_{k=0}^{p}\left\|f^{k}(x)\right\|^{2}<(p+1) \widetilde{R}^{2}$, therefore, there exists $k \in\{0,1, \ldots, p\}$ such that $\left\|f^{k}(x)\right\|<\widetilde{R}$. This implies that $f^{k+m}(x) \in B(\tilde{R})$, for any $m \geq \tilde{p}$. For $m=p-k+\tilde{p}$ we obtain $f^{p+\tilde{p}}(x) \in B(\widetilde{R})$, meaning that $x \in \widetilde{M}_{p+\tilde{p}}$.

\section{Numerical examples}

4.1. Example with known domain of attraction. Let the following discrete dynamical system be

$$
\begin{aligned}
& x_{k+1}=\frac{1}{2} x_{k}\left(1+x_{k}^{2}+2 y_{k}^{2}\right) \\
& y_{k+1}=\frac{1}{2} y_{k}\left(1+x_{k}^{2}+2 y_{k}^{2}\right)
\end{aligned} \quad k \in \mathbb{N} .
$$

There exists an infinity of steady states for this system: $(0,0)$ (asymptotically stable) and all the points $(x, y)$ belonging to the ellipsis $x^{2}+2 y^{2}=1$ (all unstable). The domain of attraction of $(0,0)$ is $D_{a}(0,0)=\left\{(x, y) \in \mathbb{R}^{2}: x^{2}+2 y^{2}<1\right\}$. 


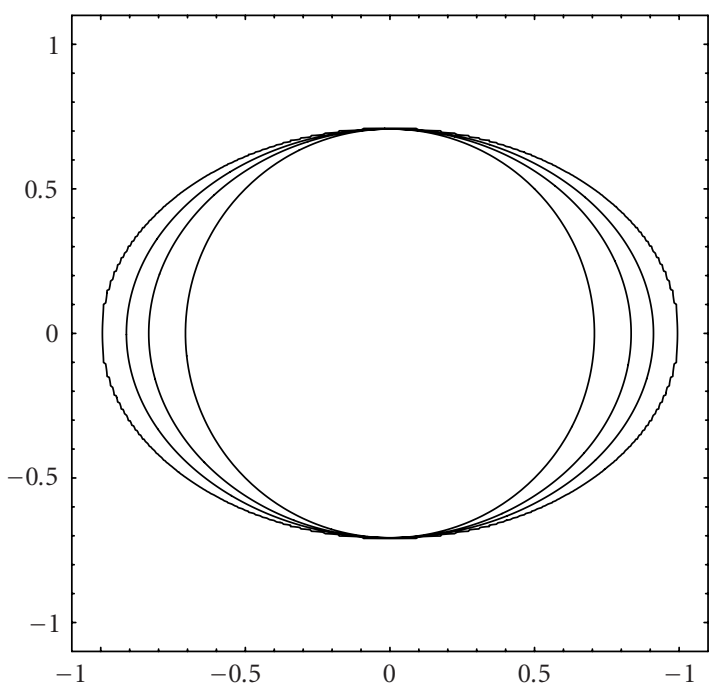

Figure 4.2. The sets $M_{p}, p=0,1,2,6$ for (4.1).

As $\left\|\partial_{(0,0)} f\right\|=1 / 2$, we compute the largest number $R>0$ such that $\|f(x)\|<\|x\|$ for any $x \in B(R) \backslash\{0\}$, and we find $R=0.7071$.

For $p=0,1,2,3,4$, we find the $N_{p}$ sets shown in Figure 4.1, parts of $D_{a}(0,0)\left(N_{p} \subset\right.$ $N_{p+1}$, for $\left.p \geq 0\right)$. In Figure 4.1, the thick-contoured ellipsis represents the boundary of $D_{a}(0,0)$.

In Figure 4.2, the sets $M_{p}$ are represented, for $p=0,1,2,6\left(M_{p} \subset M_{p+1}\right.$, for $\left.p \geq 0\right)$. Note that $M_{6}$ approximates with a good accuracy the domain of attraction.

4.2. Discrete predator-prey system. We consider the discrete predator-prey system:

$$
\begin{gathered}
x_{k+1}=a x_{k}\left(1-x_{k}\right)-x_{k} y_{k} \\
y_{k+1}=\frac{1}{b} x_{k} y_{k}
\end{gathered} \text { with } a=\frac{1}{2}, b=1, k \in \mathbb{N} .
$$

The steady states of this system are $(0,0)$ (asymptotically stable), $(-1,0)$ and $(1,-1)$ (both unstable).

We have that $\left\|\partial_{(0,0)} f\right\|=1 / 2$, and the largest number $R>0$ such that $\|f(x)\|<\|x\|$ for any $x \in B(R) \backslash\{0\}$ is $R=0.65$.

Figure 4.3 presents the $N_{p}$ sets for $p=0,1,2,3,4,5$, parts of $D_{a}(0,0)\left(N_{p} \subset N_{p+1}\right.$, for $p \geq 0)$. The black points in Figure 4.3 represent the steady states of the system.

In Figure 4.4, the sets $M_{p}$ are represented, for $p=0,1,2,6\left(M_{p} \subset M_{p+1}\right.$, for $\left.p \geq 0\right)$. Note that the boundary of $M_{6}$ approaches very much the fixed points $(-1,0)$ and $(1,-1)$, which suggests that $M_{6}$ is a good approximation of $D_{a}(0)$. 
St. Balint et al. 13

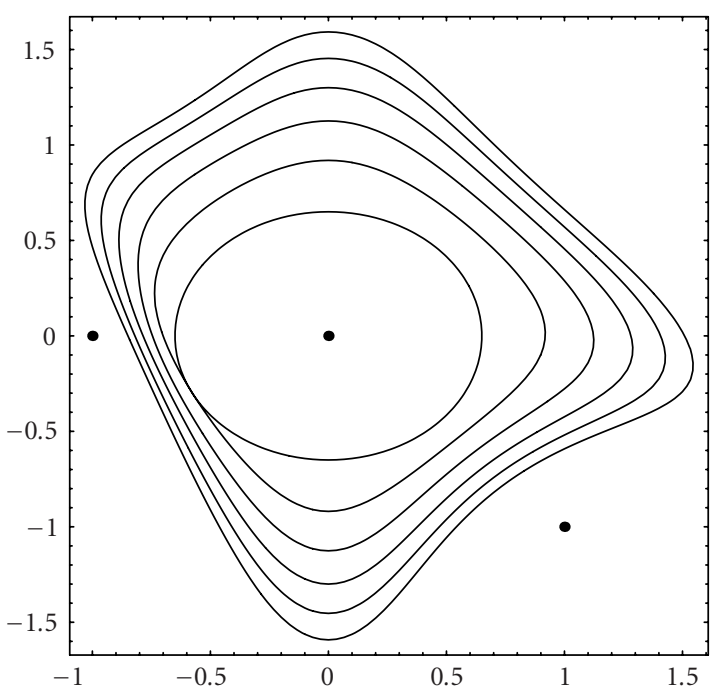

Figure 4.3. The sets $N_{p}, p=\overline{0,5}$ for (4.2).

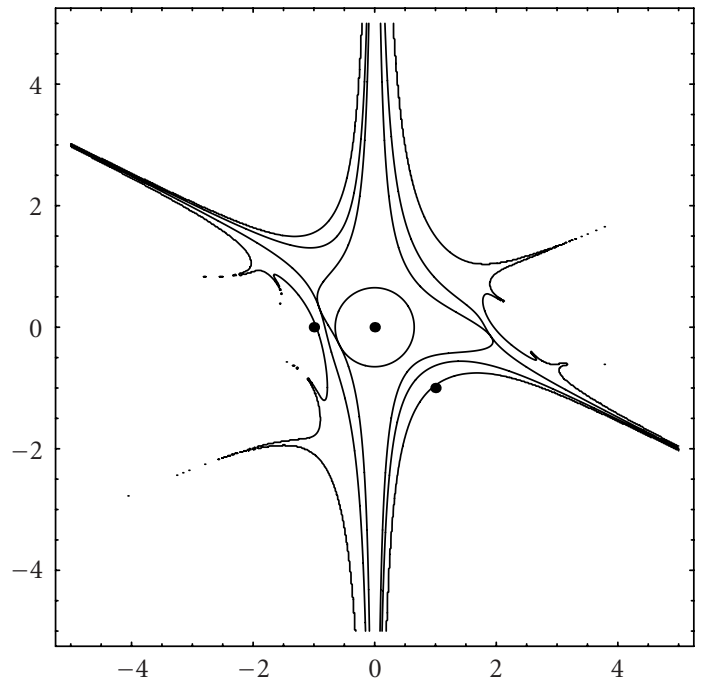

Figure 4.4. The sets $M_{p}, p=0,1,2,6$ for (4.2).

4.3. Discrete Van der Pol system. Let the following discrete dynamical system, obtained from the continuous Van der Pol system be

$$
\begin{gathered}
x_{k+1}=x_{k}-y_{k} \\
y_{k+1}=x_{k}+(1-a) y_{k}+a x_{k}^{2} y_{k}
\end{gathered} \quad \text { with } a=2, k \in \mathbb{N} .
$$




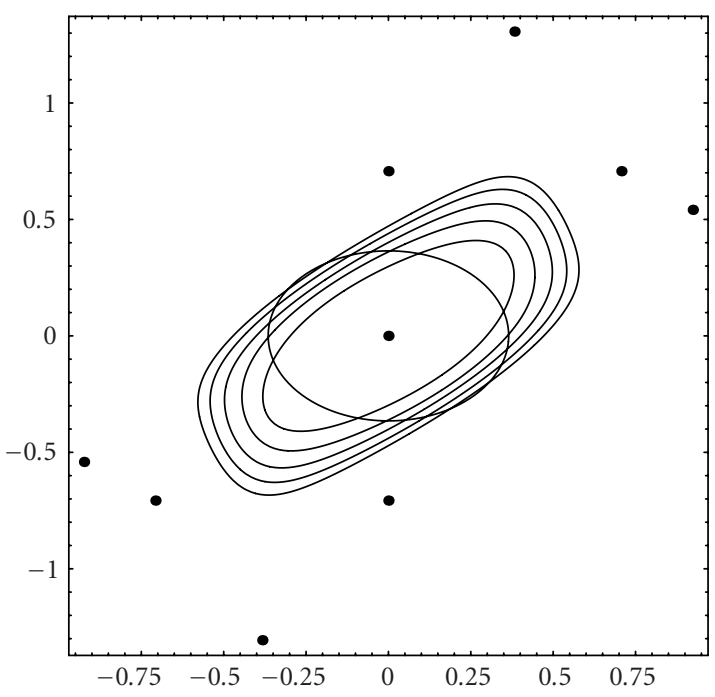

Figure 4.5. The sets $\tilde{N}_{p}, p=\overline{0,5}$ for (4.3).

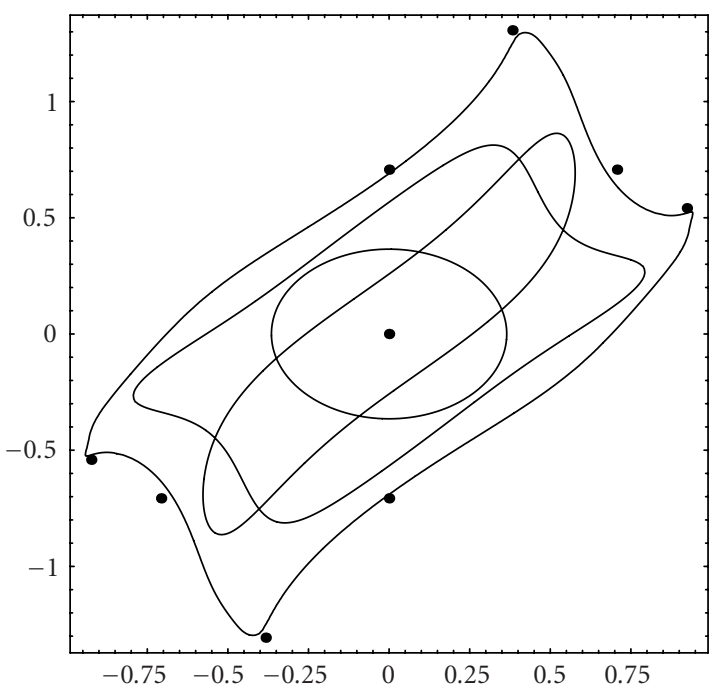

Figure 4.6. The sets $\widetilde{M}_{p}, p=0,1,2,6$ for (4.3).

The only steady state of this system is $(0,0)$ which is asymptotically stable. There are many periodic points for this system, the periodic points of order $\overline{2,5}$ being represented in Figure 4.5 by the black points.

We have that $\left\|\partial_{(0,0)} f\right\|=2$ but $\rho\left(\partial_{(0,0)} f\right)=0$. First, we observe that for $\tilde{p}=2$ we have that $\left(\partial_{(0,0)} f\right)^{\tilde{p}}=O_{2}$, therefore, $\left\|\left(\partial_{(0,0)} f\right)^{p}\right\|=0$ for any $p \geq \tilde{p}$. 
The largest number $\widetilde{R}>0$ such that $\left\|f^{p}(x)\right\|<\|x\|$ for $p \in\{\tilde{p}, \tilde{p}+1, \ldots, 2 \tilde{p}-1\}=$ $\{2,3\}$ and $x \in B(\widetilde{R}) \backslash\{0\}$ is $\widetilde{R}=0.365$.

For $p=0,1,2,3,4,5$, the connected components which contain $(0,0)$ of the $\tilde{N}_{p}$ sets are shown in Figure 4.5. We have that $\tilde{N}_{0} \nsubseteq \tilde{N}_{1} \subset \tilde{N}_{2} \subset \tilde{N}_{3} \subset \tilde{N}_{4} \subset \tilde{N}_{5}$.

In Figure 4.6 , the sets $\widetilde{M}_{p}$ are represented, for $p=0,1,2,6$. Note that the inclusions $\widetilde{M}_{p} \subset \widetilde{M}_{p+1}$ do not hold.

\section{References}

[1] R. A. Horn and C. R. Johnson, Matrix Analysis, Cambridge University Press, Cambridge, 1985.

[2] E. Kaslik, A. M. Balint, S. Birauas, and St. Balint, Approximation of the domain of attraction of an asymptotically stable fixed point of a first order analytical system of difference equations, Nonlinear Studies 10 (2003), no. 2, 103-112.

[3] E. Kaslik, A. M. Balint, A. Grigis, and St. Balint, An extension of the characterization of the domain of attraction of an asymptotically stable fixed point in the case of a nonlinear discrete dynamical system, Proceedings of 5th ICNPAA (S. Sivasundaram, ed.), European Conference Publications, Cambridge, UK, 2004.

[4] W. G. Kelley and A. C. Peterson, Difference Equations, 2nd ed., Harcourt/Academic Press, California, 2001.

[5] H. Koçak, Differential and Difference Equations through Computer Experiments, 2nd ed., Springer, New York, 1989.

[6] G. Ladas, C. Qian, P. N. Vlahos, and J. Yan, Stability of solutions of linear nonautonomous difference equations, Applicable Analysis. An International Journal 41 (1991), no. 1-4, 183-191.

[7] V. Lakshmikantham and D. Trigiante, Theory of Difference Equations. Numerical Methods and Applications, Mathematics in Science and Engineering, vol. 181, Academic Press, Massachusetts, 1988.

[8] J. P. LaSalle, The Stability and Control of Discrete Processes, Applied Mathematical Sciences, vol. 62, Springer, New York, 1986.

[9] Stability theory for difference equations, Studies in Ordinary Differntial Equations (J. Hale, ed.), MAA Studies in Mathematics, vol. 14, Taylor and Francis Science Publishers, London, 1997, pp. 1-31.

St. Balint: Department of Mathematics, West University of Timişoara, Bd. V. Parvan 4, 300223 Timişoara, Romania

E-mail address: balint@balint.math.uvt.ro

E. Kaslik: Department of Mathematics, West University of Timişoara, Bd. V. Parvan 4, 300223 Timişoara, Romania

Current address: LAGA, UMR 7539, Institut Galilée, Université Paris 13, 99 Avenue J.B. Clément, 93430 Villetaneuse, France

E-mail address: kaslik@math.univ-paris13.fr

A. M. Balint: Department of Physics, West University of Timişoara, Bd. V. Parvan 4, 300223 Timişoara, Romania

E-mail address: balint@physics.uvt.ro

A. Grigis: LAGA, UMR 7539, Institut Galilée, Université Paris 13, 99 Avenue J.B. Clément, 93430 Villetaneuse, France

E-mail address: grigis@math.univ-paris13.fr 